\title{
An Advanced Physics of Superfluidity and Superconductivity
}

\author{
Gilbert A. Ibitola ${ }^{1}$, Olanrewaju Ajanaku ${ }^{1}$, Lawrence O. Imafidon ${ }^{2}$ \\ ${ }^{1}$ Department of Physical Sciences, Ondo State University of Science \& Technology, Okitipupa, Nigeria \\ ${ }^{2}$ Department of Physical Sciences, Yaba College of Technology, Yaba, Lagos, Nigeria
}

Email address:

ibitolaieee@gmail.com (G. A. Ibitola), lanrayus@gmail.com (O. Ajanaku), lawfidon@gmail.com (L. O. Imafidon)

\section{To cite this article:}

Gilbert A. Ibitola, Olanrewaju Ajanaku, Lawrence O. Imafidon. An Advanced Physics of Superfluidity and Superconductivity. American Journal of Modern Physics. Vol. 5, No. 6, 2016, pp. 177-183. doi: 10.11648/j.ajmp.20160506.13

Received: October 24, 2016; Accepted: November 7, 2016; Published: December 5, 2016

\begin{abstract}
This paper presents an advanced Physics of superfluidity and superconductivity. We know from Quantum Mechanics that there are two types of particles, bosons and fermions. Single states can be occupied by any number of bosons while for fermions a single state can be occupied at most by one fermion. The charged boson system is found to exhibit superfluidity: the gauge-invariant Lagrangian, coupling between the bosons and the electromagnetic gauge field. It is observed that current conservation puts constraints on current correlation. Current correlation functions and electromagnetic responses are then determined for superfluids and metals. The response function in a metallic conductor is used in obtaining its several parameters which include conductivity, dielectric constant, polarization, magnetic moment density and magnetic susceptibility. The London equation is then deduced for superconductors.
\end{abstract}

Keywords: Gauge-Invariant Lagrangian, Current Correlation, Electromagnetic Responses, London Equation, Superfluid, Metal, Superconductor, Free Electron Theory, Meissner Effect

\section{Introduction}

Historically, superfluidity was discovered some time after superconductivity. The similarity of the two words is no coincidence. A superconductor is also a phase of a system in which a charge is transported without dissipation. In a metal or alloy, this charge is electric charge, and its electric resistance that becomes unmeasurably small. Also for the vortices, there is an analogue in a superconductor: flux tubes, quasi-one-dimensional objects in which a magnetic field can penetrate the superconductor. And, as for the superfluid, there is a critical temperature above which superconductivity is lost.

A superconductor can be said to break a local symmetry spontaneously, while a superfluid breaks a global symmetry spontaneously. This statement shows the theoretical similarity of the two phenomena, but also emphasizes their only fundamental difference a superconducting metal is different from superfluid helium in many aspects. For instance, electrons are fermions, while helium atoms are bosons. A charged boson system is a superfluid in which exists coupling between the bosons and the electromagnetic gauge field as well as the existence of a conserved current. The gauge - invariant Lagrangian will be derived in Section 2 to demonstrate the coupling between current or charge carriers and the electromagnetic gauge field in superfluids. An expression for the conserved current in a superfluid will also be derived in Section 2. In Section 3, expressions for the current correlation functions and electromagnetic responses in superfluids will be deduced. The free electron model and the response function in metals will be presented in Section 4. In Section 5, the response function for the boson superfluid will be presented. The London equation will be deduced and other equations will be given for superconductors in Section 6. Table 1 presents the various critical temperatures of some element and compound superconductors. Figure 1 shows the functional relationships between metals, superfluids and superconductors. 
effects [no thermo-electricity, critical or transition-temperature dependent]

$$
\text { 1. } T_{c}(B)=T_{c \max }\left[1-\left(c_{0}\right)^{2}\right]^{\circ}
$$

2. With $B_{e x}$ applied, $B=0$ inside a superconductor.

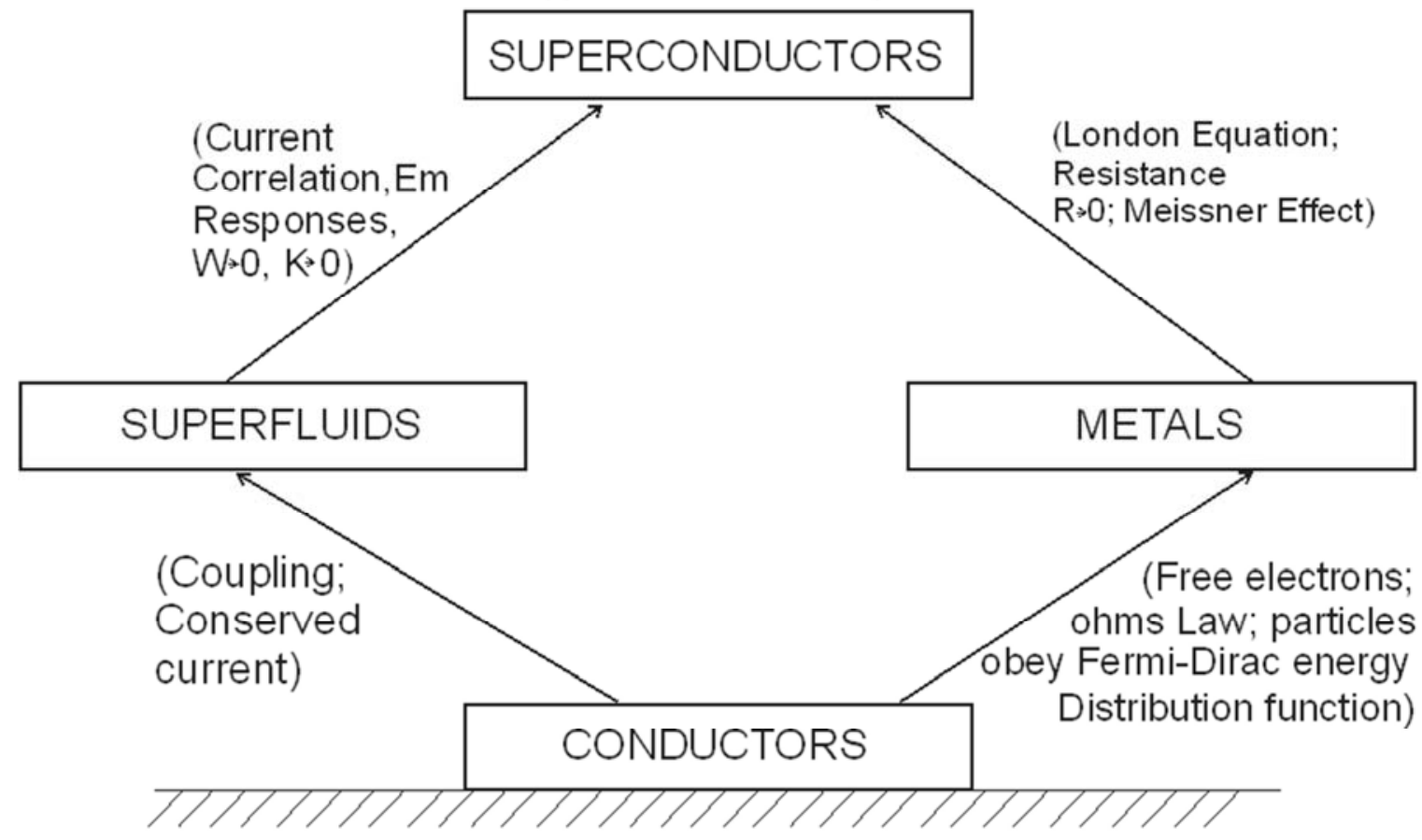

Figure 1. Relationships between superfluids, metals and superconductors.

\section{The Charged Boson Superfluid}

BOSONS are particles that obey the Bose-Einstein distribution which is given by

$$
\begin{aligned}
F_{B E}=\frac{1}{e^{\alpha} e^{E i / K T}-1} & =\frac{1}{\left[e^{(E i-E F) / K T}-1\right]} \\
& =\frac{d n_{E}}{g(E) \cdot d E}
\end{aligned}
$$

Where: $\mathrm{E}_{\mathrm{F}}=$ Fermi Energy;

$g(E)=$ density of states within energy interval $d E$;

$\mathrm{dn}_{\mathrm{E}}=$ number of bosons having energy between $\mathrm{E}$ and $\mathrm{E}+$ $\mathrm{dE}$;

$\propto=$ characteristics ratio of the system of bosons $=0$ (for bosons).

Bosons are identical and indistinguishable, possess integer spins, and do not obey Pauli Exclusion Principle. Examples of boson systems are light photons, hydrogen $\mathrm{H}_{2}$ molecules, and liquid helium atoms. When a boson system is charged and cooled down to or below its critical temperature $T_{C}$, it becomes a superfluid. The boson system superfluid can be analyzed using a theory with global U(I) symmetry which contains a conserved charge and can be coupled to a U(I) gauge field. The electromagnetic vector potential is a $\mathrm{U}(\mathrm{I})$ gauge field.

A charged boson system couples to an electromagnetic gauge field. In the presence of a non-zero electromagnetic field, the Lagrangian for a charged boson system (of boson mass $\mathrm{m}$ ) needs to be modified. It is known that the boson system Lagrangian

$$
\begin{gathered}
L(\Psi)=i\left(\frac{1}{2}\right)\left(\Psi^{*} \delta_{t} \Psi-\Psi \delta_{t} \Psi^{*}\right)-\frac{1}{2 m} \delta_{x} \Psi^{*} \delta_{x} \Psi+\mu|\Psi|^{2}- \\
\frac{V_{o}}{2}|\Psi|^{*}
\end{gathered}
$$

Is invariant (does not vary) under a global U(I) transformation

$$
\Psi \rightarrow e^{i f} \Psi
$$

i.e., that $L\left(e^{i f} \Psi\right)=\mathrm{L}(\Psi)$. However, it is not invariant (does vary) under a local $\mathrm{U}(\mathrm{I})$ transformation

$$
\Psi(\mathrm{x}, \mathrm{t}) \rightarrow e^{i f(x, t)} \Psi(\mathrm{x}, \mathrm{t}) .
$$

We have:

$$
\begin{gathered}
L\left(e^{i f(x, t)} \Psi\right)=\mathrm{i}\left(\frac{1}{2}\right)\left(\left(\Psi^{*}\left(\partial_{o}+i \partial_{o} f\right) \Psi-\Psi\left(\partial_{o}+\right.\right.\right. \\
\left.\left.\left.i \partial_{o} f\right) \Psi^{*}\right)-\frac{1}{2 m}\left|\left(\partial_{i}+i \partial_{i} f\right) \Psi\right|^{2}+\mu|\Psi|^{2}-\frac{V_{o}}{2}|\Psi|^{4}\right)
\end{gathered}
$$

Where the subscript $\mathrm{O}$ indicates the time direction and the subscript $i=1,2,3, \ldots, d$ represents the spatial directions. Let us use the Greek letter $\mu, v$, etc. to represent space- time directions. For example, $x^{\mu}$ represents the x-axis space-time coordinates. The coupling between the bosons and the electromagnetic gauge field can now be obtained by replacing $\partial_{\mu} f$ by $A_{\mu}$ as follows

$$
\begin{aligned}
\mathrm{L}\left(\Psi, A_{\mu}\right)= & \mathrm{i}\left(\frac{1}{2}\right)\left(\Psi^{*}\left(\partial_{o}+i A_{0}\right) \Psi-\Psi\left(\partial_{o}+i A_{0}\right) \Psi^{*}\right)- \\
& \frac{1}{2 m}\left|\left(\partial_{i}+i A_{i}\right) \Psi\right|^{2}+\mu|\Psi|^{2}-\frac{V_{o}}{2}|\Psi|^{4}
\end{aligned}
$$

The following Lagrangian has an interesting property in 
that it is gauge- invariant:

$$
\begin{gathered}
\Psi \rightarrow \widetilde{\Psi}=e^{i f(x, t)} . \Psi A_{\mu} \rightarrow \widetilde{A_{\mu}}=A_{\mu}-\partial_{\mu} f \\
\mathrm{~L}\left(\Psi, A_{\mu}\right) \rightarrow L\left(\widetilde{\Psi}, \widetilde{A_{\mu}}\right)=\mathrm{L}\left(\Psi, A_{\mu}\right)
\end{gathered}
$$

In equation (6), we have:

$$
\partial_{\mu} f=A_{\mu}+g \partial_{v} F v \mu=A_{\mu}+g \partial_{v}\left(\partial_{\mu} A_{v}-\partial_{v} A_{\mu}\right)
$$

Where: $A_{\mu}=$ gauge potential (or field)

$F v \mu=$ Field strengths of $A_{\mu}$.

Equation (5) only describes the coupling between the charge field $\Psi$ and the gauge field $A_{\mu}$. Hence, the complete gauge-invariant Lagrangian that describes the dynamics of both $\Psi$ and $A_{\mu}$ in the superfluid consisting of interacting charged boson system and electromagnetic field is given by:

$$
\mathrm{L}\left(\Psi, A_{\mu}\right)=\mathrm{i}\left(\frac{1}{2}\right)\left(\Psi^{*}\left(\partial_{o}+i A_{0}\right) \Psi-\Psi\left(\partial_{o}+i A_{0}\right) \Psi^{*}\right)-
$$

$\frac{1}{2 m}\left|\left(\partial_{i}-i A i\right) \Psi\right|^{2}+\mu|\Psi|^{2}-\frac{V_{o}}{2}|\Psi|^{4}+\frac{1}{\left(8 \pi e^{2}\right)}\left(\frac{1}{c}(E)^{2}-c B^{2}\right)(9)$

Where: $\mathrm{c}=$ speed of light;

$E_{i}=$ Electric field of the $\mathrm{U}(\mathrm{I})$ gauge theory $=\partial_{o} A_{i}-$ $\partial_{i} A_{0}=F_{o i}$;

$B_{i}=$ magnetic field of the $\mathrm{U}(\mathrm{I})$ gauge theory $=\varepsilon_{i j k} \partial_{j} A_{k}=$ $\frac{1}{2}\left(\varepsilon i j k F_{j k}\right)$

It is known that the considered scientific model with a global U (I) symmetry has a conserved charge. The gauged action is gauge-invariant:

$$
\mathrm{S}\left(\Psi, A_{\mu}\right)=S\left(e^{i f(x, t)} \Psi, A_{\mu}-\partial_{\mu} F\right)
$$

Let $\Psi_{c}(x, t)$ be a solution of the classical equation of motion. Then

$$
S\left(e^{i f(x, t)} \Psi_{c}, A_{\mu}\right)=S\left(\Psi_{c}, A_{\mu}\right)+O\left(f^{2}\right)
$$

Therefore:

$$
\begin{gathered}
S\left(\Psi_{c}, A_{\mu}\right)=S\left(\Psi_{c}, A_{\mu}-\partial_{\mu} f\right)+O\left(f^{2}\right) \\
=S\left(\Psi_{c}, A_{\mu}\right)+\int d^{d} x d t \partial_{\mu} f J^{\mu}\left(\Psi_{c}, A_{\mu}\right)+O\left(f^{2}\right)
\end{gathered}
$$

Where $J^{\mu}$ is the current which is equal to:

$$
J^{\mu}\left(\Psi, A_{\mu}\right)=-\partial_{A_{\mu}} L\left(\Psi, A_{\mu}\right)
$$

It can be seen that if $\Psi(x, t)$ satisfies the classical equation of motion, then

$\int d^{d} x d t . \partial_{\mu} f J^{\mu}\left(\Psi_{c}, A_{\mu}\right)=0$ (for any $f$ ) and the current $J^{\mu}\left(\Psi_{c}, A_{\mu}\right)$ is conserved. That is, we have:

$$
\partial_{\mu} J^{\mu}\left(\Psi_{c}, A_{\mu}\right)=\partial_{t} \rho+\partial_{t} \rho+\partial_{i} J^{i}=0
$$

Where $\rho=J^{o}$ is the density and $J^{i}$ is the current.

Equation (13) is also true for a zero $A_{\mu}$ field, such that:

$$
\partial_{\mu} J^{\mu}\left(\Psi_{c}\right)=0
$$

This shows current conservation in a neutral boson system. For a charged boson system, the conserved current is given by:

$$
\begin{gathered}
J^{o}=\rho=\Psi^{*} \Psi \\
J^{i}=\frac{-i}{2 m}\left[\Psi^{*}\left(\partial_{i} \Psi\right)-\left(\partial_{i} \Psi^{*}\right) \Psi\right]+A_{i}|\Psi|^{2}
\end{gathered}
$$

It is interesting to see that the current in a charged boson depends spatially on the gauge potential. Thus, a superfluid conducts electricity without any resistance, couples with the existing electromagnetic gauge field and possesses a conserved current.

\section{Current Correlation Functions and Electromagnetic Responses in Superfluids}

Current conservation puts constraints on current correlations. Many physical quantities, such as compressibility and conductivity, are determined by current correlations. To obtain the correct responses, it is important to take the limits, wave number vector $\mathrm{K} \rightarrow 0$ and the angular frequency $w \rightarrow 0$, in the appropriate order. Now let us find the response of the superfluid system to an external gauge potential. Let us find how much current $J^{\mu}$ the gauge potential $A_{\mu}$ can generate.

Introducing $J^{\mu}$ according to

$$
j^{o}=\rho ; j^{i}=\frac{-i}{2 m}\left[\Psi^{*}\left(\partial_{i} \Psi\right)-\left(\partial_{i} \Psi^{*}\right) \Psi\right],
$$

it can be seen that the Lagrangian has the form:

$$
L\left(\Psi, A_{\mu}\right)=L(\Psi)-A_{o} J^{O}-A_{i} J^{i}-\frac{1}{2 m} \rho\left(A^{i}\right)^{2}
$$

Using the linear response theory to find $\left\langle j^{\mu}(x, t)\right\rangle$ to leading order in $A_{\mu}$, we obtain the current $J^{\mu} \equiv-\partial_{A_{\mu}} L$ :

$$
\begin{aligned}
& \left\langle J^{\mu}(x, t)\right\rangle=\left\langle j^{\mu}(x, t)\right\rangle+\left(1-\partial^{\mu o}\right) A^{\mu} \cdot \rho \\
& =\int d^{d} x d t \pi^{\mu v}\left(x, t ; x^{I}, t^{I}\right) A_{v}\left(x^{I}, t^{I}\right)
\end{aligned}
$$

For equation (18), the response functions are given by:

$$
\begin{gathered}
\pi^{00}\left(x, t ; x^{l}, t^{i}\right)=-i \theta\left(t-t^{l}\right)<\left[\rho(x, t), \rho\left(x^{l}, t^{i}\right)\right] \\
\pi^{0 i}\left(x, t ; x^{l}, t^{i}\right)=-i \theta\left(t-t^{l}\right)<\left[\rho(x, t), j^{i}\left(x^{l}, t^{i}\right)\right] \\
\pi^{i 0}\left(x, t ; x^{l}, t^{i}\right)=-i \theta\left(t-t^{l}\right)<\left[j^{i}(x, t), \rho\left(x^{l}, t^{i}\right)\right] \\
\pi^{i j}\left(x, t ; x^{l}, t^{i}\right)=-i \theta\left(t-t^{l}\right)<\left[j^{i}(x, t), j^{j}\left(x^{l}, t^{i}\right)\right]+ \\
\varepsilon^{i j} \partial\left(x-x^{l}\right) \partial\left(t-t^{l}\right)\left\langle\frac{\rho}{m}\right\rangle \quad(19 \mathrm{a}-\mathrm{d})
\end{gathered}
$$

Due to the $A_{i}$ dependence of the current, we have an extra contact term $\partial^{i j} \partial\left(x-x^{l}\right) \partial\left(t-t^{l}\right) \frac{\langle\rho\rangle}{m}$.

If we introduce the correlation function, $\pi^{\mu v}\left(x, t ; x^{l}, t^{l}\right)=$ $-i \theta\left(t-t^{l}\right)<\left[j^{\mu} x, t ; j^{v}\left(x^{l}, t^{l}\right)\right]>$, then we have:

$$
\pi^{\mu v}=\pi^{\mu v}+\partial^{\mu v}\left(1-\partial^{0 \mu}\right)\left(1-\partial^{0 v}\right) \partial\left(x-x^{l}\right) \partial(t-
$$

The above result applies for both zero and finite 
temperatures. It is noted that:

$$
\left(\pi^{\mu v}\left(x, t ; x^{l}, t^{l}\right)\right)^{*}=\pi^{\mu v}\left(x^{l}, t^{l} ; x, t\right)
$$

or, in the w-k space,

$$
\left(\pi_{(k \lambda)}^{\mu v}\right)^{*}=\pi_{(-k \lambda)}^{\mu v}
$$

Where $K_{O}=\omega$.

Due to current conservation, the components of $\pi^{\mu v}$ are not all independent. We have, in the w-k space,

$$
K_{\mu} \pi_{(k \lambda)}^{\mu v}=0
$$

Thus, the full $\pi^{\mu v}$ can be determined from $\pi^{i j}$ as follows:

$$
\begin{gathered}
\pi_{(k \lambda)}^{o i}=\left(\pi_{(-k \lambda)}^{i o}\right)^{+}=\frac{-K j \pi_{(k \lambda)}^{j i}}{w} \\
\pi_{(k \lambda)}^{o o}=\frac{-k j}{\omega} \pi_{(k \lambda)}^{o j}=\frac{k_{i} k_{j}}{\omega^{2}} \pi_{(k \lambda)}^{i j}
\end{gathered}
$$

For a rotationally invariant system, we can further decompose $\Pi^{i j}$ and $\pi^{i j}$ into the longitudinal components, $\Pi_{k \lambda}^{11}$ and $\pi_{k \lambda}^{11}$, and the transverse components, $\Pi_{k \lambda}^{\perp}$ and $\pi_{k \lambda}^{\perp}$, respectively as follows:

$$
\begin{gathered}
\Pi_{(k \lambda)}^{i j}=\frac{k_{i} k_{j}}{K^{2}} \Pi_{(k \lambda)}^{I I}+\left(\partial_{i j}-\frac{k_{i} k_{j}}{K^{2}}\right), \Pi_{(k \lambda)}^{\perp} \\
\pi_{(k j)}^{i j}=\frac{k_{i} k_{j}}{K^{2}} \pi_{(k \lambda)}^{I I}+\left(\partial_{i j}-\frac{k_{i} k_{j}}{K^{2}}\right), \Pi_{(k \lambda)}^{\perp}
\end{gathered}
$$

Equations (22a) and (22b) can now be written as:

$$
\begin{gathered}
\Pi_{(k \lambda)}^{o i}=-K_{i} \Pi_{(k \lambda)}^{I I} ; \\
\Pi_{(k \lambda)}^{00}=\frac{K^{2}}{\omega^{2}} \Pi_{(k \lambda)}^{I I} .
\end{gathered}
$$

Equation (20) can be written as:

$$
\begin{aligned}
& \Pi_{(k \lambda)}^{I I}=\pi_{(k \lambda)}^{I I}+\frac{\langle\rho\rangle}{m} ; \\
& \Pi_{(k \lambda)}^{\perp}=\pi_{(k \lambda)}^{\perp}+\frac{\langle\rho\rangle}{m} .
\end{aligned}
$$

\section{The Free Electron Model and Applications of Response Function in Mettallic Conductors}

The postulates of the free electron theory of metallic conductors are:

[i] A metallic conductor is composed of weakly bound valence electrons of the metal's lattice atoms.

[ii] The free electrons in the metal continuum are not bound to any particular atoms but move throughout the entire solid.

[iii] Each electron in the metal experiences no net force.

[iv] Each free electron in a metal moves throughout the metal under a constant electrostatic potential.

[v] At the boundaries of the metal, the potential rises rapidly because of the net electrostatic force acting on an electron at the metal boundary.

[vi] Thus, in this free electron model, the electrons in a metal can be accurately treated like a cloud or gas of noninteracting spin $-\frac{1}{2}$ fermions confined to a three-dimensional box.

[vii] The free electrons in a metallic conductor obey the Pauli exclusion principle and the Fermi-Dirac energy distribution statistics which is given by;

$$
F_{F D}=\frac{1}{\left(e^{\propto} e^{E_{i} / K T}+1\right)}=\frac{1}{\left(e^{\left(E_{i}-E_{F}\right) / K T}+1\right)}
$$

Where: $\mathrm{E}_{\mathrm{F}}=-K T . \propto=$ Fermi Energy;

$\mathrm{K}=$ Boltzmann constant;

$\mathrm{T}=$ Absolute temperature.

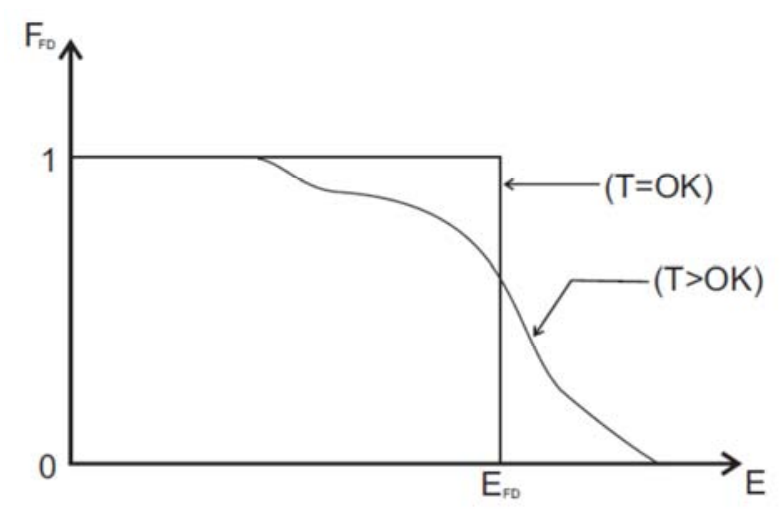

Figure 2. Plots of $F_{F D}$ Versus Energy $E$ for $T=O K$ and for $T>O K$ for a metal.

Figure 2 shows the graph of $\mathrm{F}_{\mathrm{FD}}$ against energy $\mathrm{E}$ which has a form shown by the solid curve for $\mathrm{T}=0 \mathrm{~K}$. For $\mathrm{T}>0$ $\mathrm{K}$, the graph takes on the form given by the dashed curve.

For a metallic conductor, the number of states, dS, with energies between $\mathrm{E}$ and $\mathrm{E}+\mathrm{dE}$ is:

$$
d S=\frac{\pi}{2} N^{2} d N=\frac{2 \pi V(2 m)^{3 / 2}}{h^{3}} E^{\frac{1}{2}} d E
$$

Where $\mathrm{h}=$ Planck's constant;

$\mathrm{m}=$ mass of an electron;

$\mathrm{V}=$ volume of the metallic conductor;

$\mathrm{E}=$ energy.

The density of states in the energy interval $\mathrm{dE}$ is:

$$
g(E)=\frac{2 \pi V(2 m)^{3 / 2}}{h^{3}} E^{\frac{1}{2}}
$$

The Fermi energy (at $\mathrm{T}=0 \mathrm{~K}$ ) for a metallic conductor is given by:

$$
E_{F O}=\frac{h^{2}}{8 m}\left(\frac{3 N}{\pi V}\right)^{2 / 3}
$$

Where $N / V=$ number of electrons per unit volume.

At this point let us demonstrate how the response function $\Pi^{\mu v}$ is related to many important physical quantities. Let us consider a metal in this case. In the $w \rightarrow 0$ limit, we have:

$$
\partial_{\rho}(k)=\Pi_{(o, k)}^{o o} A_{o}(k)
$$


It is noteworthy to assert that $A_{o}(k)$ is the external potential and $-\Pi_{(o, k)}^{o o}$ is the compressibility (at wave vector K). Hence,

$$
\chi(k)=-\Pi_{(o, k)}^{o o}
$$

Which is finite for all $\mathrm{K}$. Thus, for $w \rightarrow 0$, small $w$ and $w \ll k$ limits, we have:

$$
\Pi_{(k \lambda)}^{I I}=-\chi(k) \cdot w^{2} / k^{2}
$$

From equations (25a) and (25b), it can be seen that in the $w \rightarrow 0$ limit, $\pi_{(k \lambda)}^{I I}$ must exactly cancel $\langle\rho\rangle / m$ in order for $\Pi_{(k \lambda)}^{I I}$ to vanish like $\omega^{2}$.

In the $k \rightarrow 0$ limit as well as in the $|K| \ll w$ limit, the quantity $-i w A_{i},(w, k)$ is an almost uniform electric field, which is expected to generate a current that has a direction given by $A_{i}$ and a wave vector represented by $\mathrm{K}$ :

$$
J^{i}(w)=\lim _{K \rightarrow 0} \frac{\Pi^{i j}}{-i w}(-i w) A_{j}(w, k)
$$

From equations (23a) and (23b), we see that the limit $k \rightarrow 0$ exists only when $\Pi_{(k \lambda)}^{I I}=\Pi_{(k \lambda)}^{\perp}$ in the limit of $w \gg|K|$. Should this be the case, then we obtain, for the metallic conductor, the conductivity

$$
\sigma(w)=\frac{\Pi_{(w, O)}^{I I}}{(-i w)}=\frac{\Pi_{(w, 0)}^{\perp}}{(-i w)}
$$

The real part of the conductivity,

$$
\operatorname{Re\sigma }(w)=-\operatorname{Im} \frac{\Pi_{(w, o)}^{I I}}{w}=-\operatorname{Im} \frac{\Pi_{(w, 0)}^{\perp}}{w}
$$

Corresponds to energy dissipation. The imaginary part of the conductivity gives us the dielectric constant of the metallic conductor

$$
\varepsilon(w)=\frac{-I m \sigma(w)}{w}=\operatorname{Re} \frac{\Pi_{(w, o)}^{I I}}{w^{2}}=\operatorname{Re} \frac{\Pi_{(w, o)}^{\perp}}{w^{2}}
$$

Thus, in the $w \gg|k|$ limit, we have:

$$
\Pi_{(w, o)}^{I I}=\Pi_{(w, o)}^{\perp}=i w \operatorname{Re\sigma }(w)+w^{2} \varepsilon(w)
$$

If $A_{i}=0$, then the $\rho$ polarization vector $\mathrm{P}$ satisfies

$$
\begin{gathered}
\partial_{x} . P=-\partial \rho \Rightarrow i K_{i} P^{i}=-\delta \rho=-\pi^{00} A_{o}= \\
\frac{-k^{2}}{w^{2}} \Pi_{(k \lambda)}^{I I} A_{o}=\frac{i \Pi_{(k \lambda)}^{I I}}{w^{2}} K_{i} E_{i} \quad(38) \quad \text { Therefore: } \\
\pi^{o o}=\chi^{2}(-i w)(i w)\left\langle\theta_{(w, k)} \theta_{(-w,-k)}\right\rangle=\frac{\chi w^{2}}{\left(w^{2}-v^{2} k^{2}+i O^{+} \operatorname{sgn}(w)\right)} \\
\pi^{o i}=\pi^{i o}=\chi^{2}(-i w)\left(-i K_{i}\right)\left\langle\theta_{(w, k)} \theta_{(-w,-k)}\right\rangle=\frac{\chi v^{2} w K_{i}}{\left(w^{2}-v^{2} k^{2}+i 0^{+} \operatorname{sgn}(w)\right)} \\
\pi^{i j}=\chi^{2}\left(i K_{i}\right)\left(-K_{j}\right)\left\langle\theta_{(w, k)} \theta_{(-w,-k)}\right\rangle=\frac{\chi v^{4} K_{i} K_{j}}{\left(w^{2}-v^{2} k^{2}+i O^{+} \operatorname{sgn}(w)\right)}
\end{gathered}
$$

Therefore, we get:

$$
P^{i}=\frac{\Pi_{(k \lambda)}^{I I}}{w^{2}} E_{i}
$$

And it can be seen again that the dielectric constant,

$$
E(w)=\Pi_{(k \lambda)}^{I I} / w^{2} .
$$

We have so far considered the $w \gg|k|$ limit of $\Pi^{I I}$ and $\Pi^{\perp}$, as well as the $w \ll|k|$ limit of $\Pi^{I I}$. It remains the $w \ll|k|$ limit of $\Pi^{\perp}$ that is to be considered. This limit corresponds to magnetic susceptibility.

The magnetic moment density $\mathrm{M}$ satisfies

$$
\partial_{x} \times M=-j
$$

Thus, if $A_{o}=0$, we have:

$$
\begin{aligned}
i & \in^{i j k} K_{j} M_{k}=-j^{i}=-\Pi^{i j} A_{j}=-\left(\partial^{i j} K^{2}-K^{i} K^{j}\right) A_{j} \frac{\Pi_{(k \lambda)}^{\perp}}{K^{2}} \\
& =+\epsilon^{i j^{I} k^{I}} K_{i^{I}} \in^{k^{I^{I}} I_{j}} K_{i^{I}} A_{j} \frac{\Pi_{(k \lambda)}^{\perp}}{K^{2}}=-i \epsilon^{i j k} K_{j} B_{K} \frac{\Pi_{(k \lambda)}^{\perp}}{K^{2}}
\end{aligned}
$$

Therefore:

$$
M_{i}=-\frac{\Pi_{(k \lambda)}^{\perp}}{K^{2}} B_{i}
$$

And it can be seen that the proportionality constant of equation $\left(420,-\frac{\Pi_{(k \lambda)}^{\perp}}{K^{2}}\right.$, is the magnetic susceptibility of metallic conductor.

\section{Response Function of a Superfluid}

In this section, let us find the expressions or formulas for the boson superfluid. Let us, in this regard, consider the boson system superfluid Lagrangian with the gauge field (Equation 5). We integrate out the amplitude fluctuations and obtain an XY-model with a gauge field. To a quadratic order in $\left(\partial_{\mu}, \theta, A_{\mu}\right)$, we have:

$$
\left.L=\frac{\chi}{2}\left(\left(\partial_{o} \theta+A_{o}\right)^{2}\right)-v^{2}\left(\partial_{i} \theta+A_{i}\right)^{2}\right)
$$

It can be seen that:

$$
\begin{gathered}
j^{0}=-\chi \partial_{o} \theta, \\
j^{i}=\chi v^{2} \partial_{i} \theta .
\end{gathered}
$$

The use of $\mathrm{iO}^{+} \operatorname{sgn}(\mathrm{w})$ gives us the response function in each equation (equations (44a-c)). The total response function, $\Pi^{\mu v}$, in a superfluid has the following components:

$$
\Pi^{o o}=\chi\left(\frac{w^{2}}{\left(w^{2}-v^{2} k^{2}+i O^{+} \operatorname{sgn}(w)\right)}-1\right)
$$




$$
\begin{array}{r}
\Pi^{o i}=\Pi^{i o}=\frac{-\chi v^{2} w K_{i}}{\left[w^{2}-v^{2} k^{2}+i O^{+} \operatorname{sgn}(w)\right]} \\
\Pi^{i j}=\chi v^{2}\left[\partial_{i j}+\frac{v^{2} K_{i} K_{j}}{\left(w^{2}-v^{2} k^{2}+i O^{+} \operatorname{sgn}(w)\right)}\right] \\
\text { And } \Pi^{I I}=\chi v^{2}\left[\frac{v^{2} K^{2}}{\left(w^{2}-v^{2} k^{2}+i O^{+} \operatorname{sgn}(w)\right)}+1\right] \\
\Pi^{\perp}=\chi v^{2}
\end{array}
$$

\section{The London Equation and Superconductivity}

The compressibility, $-\Pi_{(o, k)}^{o o}$, is finite and is equal to $\chi$. The magnetic susceptibility of a metal conductor $-\Pi^{\perp} / c K^{2}$ diverges as $\mathrm{K} \rightarrow 0$. The real part of the conductivity,

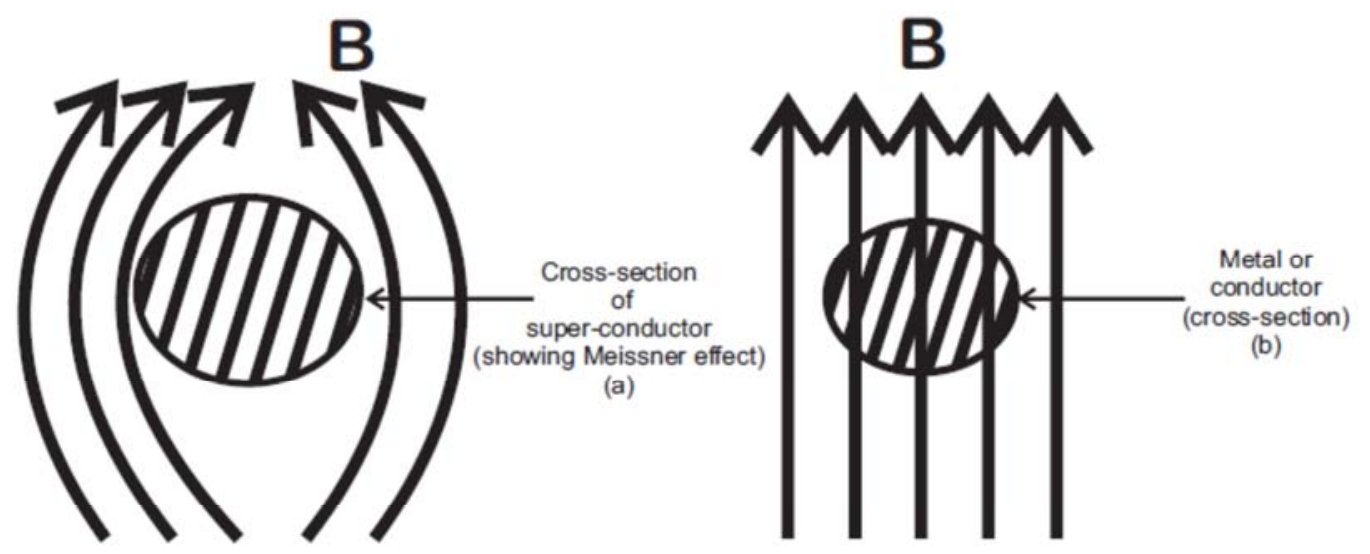

(Illustration of Meissener Effect)

Figure 3. Externally applied magnetic field lines to: (A) superconductor; (B)conductor.
For a Superconductor, we have:

$$
T=T_{c}\left[1-\left(B / B_{c}\right)^{2}\right]^{\frac{1}{2}} ; B=B_{c}\left[1-\left(T / T_{c}\right)^{2}\right]^{\frac{1}{2}} ;
$$

Where: $\mathrm{T}=$ Surrounding/Ambient Temperature;

$\mathrm{B}=$ externally applied magnetic field;

$\mathrm{T}_{\mathrm{C}}=$ Critical or transition temperature; (a characteristic temperature)

Figure 3 shows an illustration of Meissner effect in superconductors. The Meissner effect cannot be observed in metallic conductors such as copper. At a certain constant absolute temperature $\mathrm{T}$, an external magnetic field $\mathrm{B}$ is being applied to the superconductor. As the value of $\mathrm{B}$ increases, the field lines tend toward the boundary or periphery of superconductor. As $B$ is increased to a critical value $\left(B_{C}\right)$, the field lines are ejected from inside (the cross section of) the superconductor. Thus, $\mathrm{B}_{\mathrm{C}}$ is that value of an externally applied magnetic field above which there are no any field lines passing through the superconductor. This is known as Meissner effect. For a superconductor, we have:

$$
B=B_{c}\left[1-\left(T / T_{c}\right)^{2}\right]^{\frac{1}{2}}
$$

$\mathrm{B}_{\mathrm{C}}=$ critical magnetic field applied (when $\mathrm{B}=0$ inside);

$$
\operatorname{Re} \sigma(w)=\operatorname{lm} \frac{\Pi_{(w, o)}^{I I}}{w}=\operatorname{lm} \frac{x v^{2}}{\left(w+i O^{+}\right)}=\frac{\pi \rho \partial(w)}{m}
$$

Is zero for finite frequency. If we choose the coulomb gauge $\partial_{x} A=0$, then, from $J^{i}=\Pi^{i j} A_{j}$, we find a relationship between the current and the gauge potential:

$$
J=\Pi^{\perp} A_{j}=(\rho / m) A
$$

Equation (47) is the famous London equation for superconductors. It is responsible for many novel properties of superconductors such as persistent current, the Meissner effect, etc.
It is observed also that:

$$
T=T_{c}\left[1-\left(B / B_{c}\right)^{2}\right]^{\frac{1}{2}}
$$

Where: $\mathrm{T}=$ surrounding or ambient temperature $(\mathrm{K})$;

$\mathrm{T}_{\mathrm{C}}=$ critical or transition temperature $(\mathrm{K})$;

$\mathrm{B}=$ externally applied magnetic field $\left(\mathrm{Wb} / \mathrm{m}^{2}\right) ; \mathrm{B}_{\mathrm{c}}=$ critical value of $B$.

A metallic conductor obeys Ohm's law:

$$
V=I \cdot R
$$

This equation is valid as long as $\mathrm{R}$ is finite.

If $\mathrm{R}$ is infinitely large, we get an insulator. If $\mathrm{R}$ tends to zero, so that the current is infinitely large, we obtain a superconductor. There are some elements or compounds which exhibit superconductivity as long as the ambient temperature $(\mathrm{T})$ of the chosen material is maintained at the critical or transition temperature $\left(\mathrm{T}_{\mathrm{C}}\right)$ of that material.

\section{Conclusion}

In this paper, we have derived gauge-invariant Lagrangian for a superfluid which demonstrates the coupling between the charged superfluid particles and the electromagnetic gauge 
field. It also shows that there is a conserved current in the superfluid field.

Current correlation functions and electromagnetic response functions are found for a superfluid. Current conservation puts constraint on current correlations. Some important physical quantities for a metallic conductor, such as compressibility, dissipation, conductivity, polarization, dielectric constant and magnetic susceptibility, are obtained for the conductor. A conductor becomes a superconductor when it is subjected to the transition or critical values of an externally applied magnetic field or absolute temperature. The London equation is derived and the Meissner's effect is discussed for a superconductor.

\section{Appendix}

Table 1. Characteristics or Critical Temperature of Some Superconductors.

\begin{tabular}{llll}
\hline ELEMENT & & \multicolumn{3}{l}{ COMPOUND } \\
\multicolumn{2}{l}{ SUPERCONDUCTOR } & \multicolumn{2}{l}{ SUPERCONDUCTOR } \\
\hline ELEMENT & $\mathrm{T}_{\mathrm{C}}\left({ }^{\mathrm{O}} \mathrm{K}\right)$ & $\mathrm{COMPOUND}$ & $\mathrm{T}_{\mathrm{C}}\left({ }^{\mathrm{O}} \mathrm{K}\right)$ \\
Tungsten & 0.01 & $\mathrm{ZrAl}_{2}$ & 0.30 \\
Cadmium & 0.56 & $\mathrm{AuBe}$ & 2.64 \\
Mercury & 4.15 & $\mathrm{NiBi}$ & 4.25 \\
Niobium & 9.46 & $\mathrm{Nb}_{3} \mathrm{Al}_{0.8} \mathrm{Si}_{0.2}$ & 18.05 \\
Technetium & $7.92-8.22$ & $\mathrm{Nb}_{3} \mathrm{Al}_{0.8} \mathrm{Ge}_{0.2}$ & 20.05 \\
\hline
\end{tabular}

NOTE: Silver, Gallium, Iridium, Lead etc. are also superconductors having different critical or transition temperatures.

\section{References}

[1] Caldeira, A. O. and A. J. Leggett, 1991, Phys. Rev. Lett., 54, 411.

[2] Chaikin, P. M. and T. C. Lubensky, 2007, "Principles Of Condensed Matter Physics" (Cambridge University Press).
[3] Chang, A. M., L. N. Pfeiffer and K. W. West, 2006, Phys. Rev. Lett. 77, 2538.

[4] Frohlich, J. and U. M. Studer, 2003, Rev. Of Mod. Phys. 73, 1033.

[5] Gliozzi, F., T. Regge and M. A. Virasoro, 1999, Physics Letters B 101, 876 .

[6] Haldane, F., 2002, Helv. Phys. Acta. 75, 1153.

[7] Iso, S., D. Karabali and B. Sakita, 2002, Phys. Lett. B 306, 1267.

[8] Jain, J. K. and S. A. Kivelson, 1998, Phys. Rev. Lett. 71, 1565.

[9] Landau, L. D. and E. M. Lifschitz, 1985, Statistical Physics Vol. 5 (Pergamon, London).

[10] Ma, S. K., 1986, "Modern Theory Of critical Phenomena" (Benjamin/Cummings, Reading, MA).

[11] Sachdev, S. and K. Park, 2007, Annals Of Physics (N.Y.) 298, 58.

[12] Casalbuoni, R. 2011, Introduction to Quantum Field Theory, World Scientific Publishing, Singapore.

[13] Nazarov Y. V. and J. Danon, 2013 Advanced Quantum Mechanics, Cambridge Univ. Press.

[14] Tilley D. R. and John Tilley, 1986 Superfluidity and Superconductivity, Adam Hilger LTD, Bristol and London.

[15] Annett J., 2004, Superconductivity, Superfluids, and Condensates (Oxford Univ. Press, New York).

[16] Alford, M. G., S. K. Mallavarapu, A. Schmitt, S. Stetina, 2013, Phys. Rev. D87, 065001.

[17] He L., S. Mao, P. Zhuang, 2013 Int.J.Mod.Phys. A28, 1330054.

[18] Watanabe H., H. Murayama, 2014, eprint arXiv:1402.7066. 